BMJ Open

Sport \&

Exercise

Medicine

\title{
Ankle syndesmosis repair and rehabilitation in professional rugby league players: a case series report
}

\author{
Alex James Latham, ${ }^{1}$ Peter Charles Goodwin, ${ }^{2}$ Ben Stirling, ${ }^{3}$ Adam Budgen ${ }^{4}$
}

To cite: Latham AJ,

Goodwin PC, Stirling B, et al. Ankle syndesmosis repair and rehabilitation in professional rugby league players: a case series report. BMJ Open Sport Exerc Med 2017;3:e000175.

doi:10.1136/bmjsem-2016000175

Accepted 28 February 2017

CrossMark

${ }^{1}$ East Cheshire NHS Trust, Macclesfield District General Hospital, Macclesfield, Cheshire, UK

${ }^{2}$ Manchester Metropolitan University, Manchester, UK ${ }^{3}$ Warrington Wolves RLFC, The Halliwell Jones Stadium, Warrington, UK

${ }^{4}$ York Hospitals NHS

Foundation Trust, York, UK

Correspondence to

Dr Alex James Latham; alex. latham@nhs.net

\section{ABSTRACT}

Background/aim: The distal tibiofibular joint is described as a syndesmosis. Traditionally, severe syndesmotic injuries with diastasis have been treated surgically with screw fixation. This case series details an ankle syndesmosis tightrope repair and an accelerated rehabilitation protocol that reduces the amount of time to return to professional rugby league in the UK. The aim of this study was to describe players' journey from injury, through diagnosis to surgery, rehabilitation and return to participation, detailing time scales and methods used at each stage to highlight the change in current practice.

Methods: Players were identified via a single orthopaedic surgeon in the UK who specialises in ankle syndesmosis repair. Between January 2010 and September 2015, adult men playing full-time professional rugby league in the UK Super League with ankle syndesmosis injuries were identified.

Results: Eighteen players from six different clubs were included. The most common mechanism of injury was forced dorsiflexion/eversion. The average return to participation was 64 days (SD 17.2, range 38-108). This compares favourably to reports of between 120 and 180 days following screw fixation.

Conclusion: Ankle syndesmosis tightrope repair and an accelerated rehabilitation protocol is as safe as traditional methods. The accelerated rehabilitation protocol promotes early weight-bearing and has shown to expedite the return to sport for professional Rugby League players. It is possible to return to sport 2 months after a tightrope repair and accelerated rehabilitation, compared with 3-6 months post screw fixation. This is extremely encouraging for the professional sporting population.

\section{INTRODUCTION}

The distal tibiofibular joint is described as a syndesmosis. It comprises the tibia and fibula, the fibrous interosseous membrane (IOM) between the two bones, the anterior inferior tibiofibular ligament (AITFL), posterior inferior tibiofibular ligament (PITFL), transverse ligament (TL) and the interosseous ligament (IOL) . ${ }^{1}$ While syndesmotic injuries are described as disruption to one or more of the ligaments, ${ }^{1}$ they often occur in

\section{Summary}

Ankle syndesmosis surgery via a double tightrope repair followed by the accelerated rehabilitation protocol is as safe as the traditional procedures.

- The accelerated rehabilitation protocol promotes early weight-bearing resulting in an effective and quick route to return to sport for professional Rugby League players.

- A period of 2 months from surgery to return to sport is possible compared with 3 to 6 months postscrew fixation, which for the professional sporting population is extremely encouraging.

conjunction with ankle fractures $13 \%-50 \%$ of the time. ${ }^{23}$

Syndesmosis injuries, or 'high ankle sprains', are relatively uncommon making up between $1 \%$ and $18 \%$ of ankle ligament injuries. ${ }^{145}$ This large variation might indicate an under-reporting of these injuries, ${ }^{6}$ reflecting the difficulty identifying syndesmotic ligament damage. ${ }^{6}$ Sporting populations are more likely to suffer from syndesmotic injuries due to the forces required to damage the ligaments. ${ }^{6} 7$ Injuries are commonly sustained during activities that involve a cutting motion, or following a forced external rotation movement on a fixed foot as can occur during contact sports. ${ }^{589}$

Syndesmotic injuries are consistently associated with higher levels of disability, pain and prolonged periods out of sporting participation. $^{10-12}$ They have also been described as one of the most difficult sporting injuries to treat, ${ }^{6}$ with rehabilitation potentially taking between twice and 30 times longer than isolated lateral ligament sprains. ${ }^{13}$

Traditionally, higher grade syndesmotic injuries have been treated surgically with screw fixation being the most common method used ( $75 \%$ of the time). ${ }^{14}$ This poses problems for the athletic population as the 
screw requires removal ( $68 \%$ of the time) between 3 and 6 months post fixation, potentially delaying rehabilitation and return to sport. ${ }^{15}$ Screw fixation can lead to malreduction in up to $39 \%$ of cases, ${ }^{16}$ causing chronic instability and symptoms. An unstable syndesmosis injury requiring surgical fixation will commonly require 4-6 months before successful return to sport. ${ }^{17}$

A novel surgical approach to syndesmosis repair has been developed involving a tightrope procedure. ${ }^{18-20}$ This involves implanting two to four cortical endobuttons and looping a non-absorbable suture around them to provide semirigid fixation. ${ }^{21}{ }^{22}$ Theoretically, it has a number of advantages over traditional screw fixation: it allows for a small amount of normal biomechanical movement at the syndesmosis; it rarely requires a second operation to remove the device; it provides significantly better anatomic reduction and it allows for earlier return to weight-bearing, rehabilitation and ultimately sport. ${ }^{7} 21$ 23-25

Rehabilitation following surgery, via either screw fixation or tightrope repair is controversial with little consensus as to the optimal regimen. ${ }^{7}$ Most regimen consist of a period of immobilisation and restricted weight-bearing, progressing to restoration of range of movement, strength and proprioception, finally sportsspecific drills prior to return to competition. ${ }^{726}$ The exact make-up of each rehabilitation regimen differs from

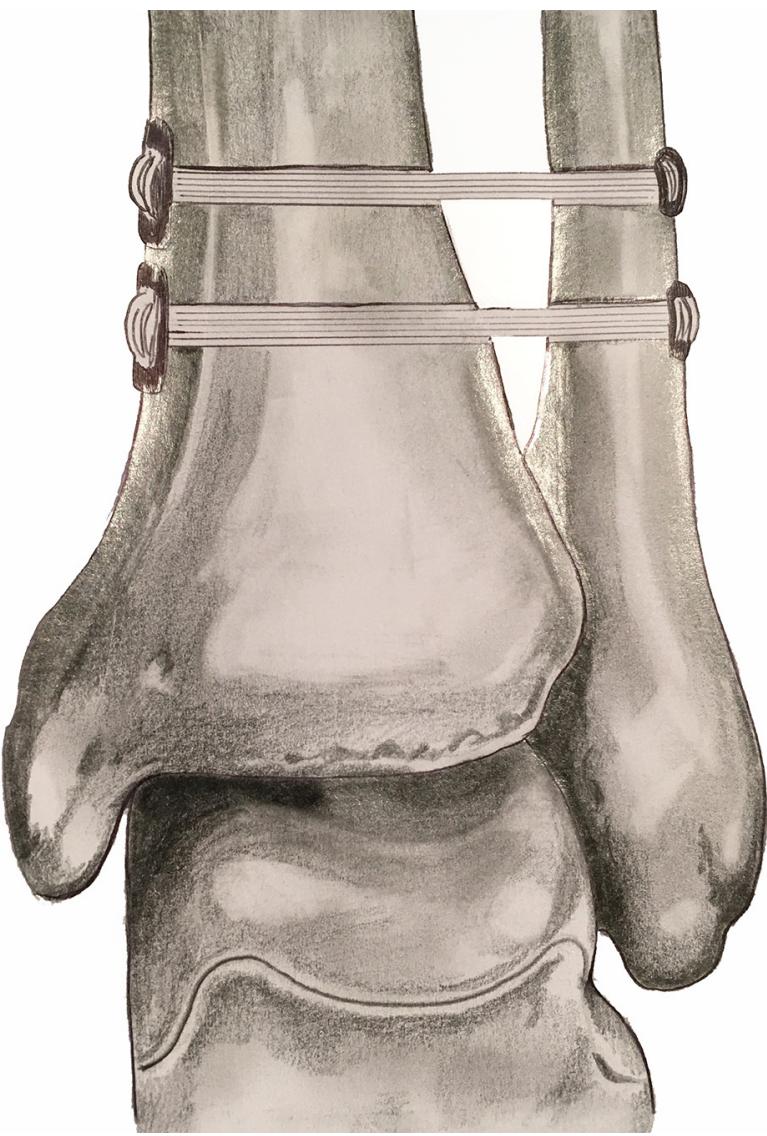

Figure 1. Syndesmosis tightrope repair. (Picture courtesy of Laura Latham, March 2016.) study to study attributed to the differing functional needs of individuals, their sporting requirements and the postsurgical protocol implemented by the surgeons.

This case series will detail an ankle syndesmosis tightrope repair and an accelerated rehabilitation protocol that significantly reduces the amount of time before return to professional rugby league in the UK is possible.

To ensure methodological rigour for the reporting of this case series report, the CARE (CAse REport consensus-based guidelines) guidelines were followed. ${ }^{27}$

\section{Patient information}

From January 2010 to September 2015, 18 adult men playing full-time professional rugby league from six different Rugby Football League (RFL) Super league clubs were identified with ankle syndesmosis injuries. Average age at the time of injury was 22 years (SD 3.3, range 17-28). Players had no current or past medical report of note and their main complaint was pain, instability and reduced ability to weight bear in the affected ankle.

Players were identified via a single orthopaedic surgeon in the UK specialising in ankle syndesmosis repair. Ethical approval for the current study was obtained from Manchester Metropolitan University. An information sheet and consent form was issued to each player detailing the current study and its aims.

Mechanism of injury, diagnostic work-up, exact surgical procedure, rehabilitation protocol followed and any adverse events data were collected. Data were collected from the therapy notes by an independent researcher $(\mathrm{AJL})$. The treating physiotherapy team were contacted to complete any omissions in the notes and to ensure accuracy of data collected.

The most common mechanism of injury reported was forced dorsiflexion/eversion $(n=8)$, isolated eversion $(n=2)$, forced rotation $(n=2)$, forced plantar flexion/ inversion $(n=3)$, insidious $(n=2)$ and one incident of forced plantar flexion accounted for the remaining cases. One player injured both ankles in separate incidents 5 months apart, otherwise all were first-time injuries.

\section{Diagnosis}

Mean time from injury to diagnosis was 21 days (SD 54.7 , range 0-210). Results were skewed by two atypical cases not being diagnosed for 3 and 7 months, respectively. Two further cases were managed conservatively for 2 weeks before diagnostic testing was initiated. With these omitted mean time from injury to diagnosis was 1.8 days (SD 2.2, range 0-7). Diagnosis was based on MRI confirmed diastasis and a physical examination by the treating surgeon.

Criteria for surgical intervention in 16 of the 18 cases was based on MRI-confirmed diastasis in keeping with the grading suggested by Sikka $e t a l^{28}$ and a surgeon- 
led approach. In the other two cases, weight-bearing $\mathrm{X}$-ray confirmed diastasis and physical examination findings were the criteria for surgery.

\section{Surgical intervention}

Average time from injury to surgery was 38.7 days (SD 73.7, range 2-265), skewed by the two atypical results. Omitting these cases, mean time from injury to surgery was 13.7 days (SD 8.73 range 2-31). Mean time from diagnosis to surgery for the group was 10 days (SD 7 range 2-22).

All players underwent a double tightrope repair performed by the same surgeon. This involved a lateral incision to provide access to the distal fibula. A $3.5 \mathrm{~mm}$ drill bit was passed through all four cortices of the tibia and fibula before being removed and the medial endobutton was passed through the predrilled hole using a needle. Once through the medial tibial cortex, the guide sutures were used to orientate the oblong medial endobutton so that it sat perpendicular to the hole flush with the bone. Arthrex TightRope (Arthrex, Naples, Florida, USA) No. 5 FiberWire (Arthrex) made of ultra-high molecular weight polyethylene anchored two metallic cortical buttons to provide physiological stabilisation of the ankle mortise (figure 1). The lateral, round endobutton was then tightened to reduce the syndesmosis and ensure the fibula sat in the fibula notch and the button sat flush with the fibula bones' outer cortex. A second tightrope was then passed in the same way to improve the stability and strength of the repair (figure 1).

Four players had a concurrent anterior talofibular ligament (ATFL) repair and one player required an open reduction internal fixation (ORIF) of the fibula. For the patient with the fractured fibula, the fracture was stabilised first using internal fixation with a plate. The tightropes were then placed through two of the empty screw holes with the lateral round endobuttons sitting flush in the screw hole recesses on the lateral side.

\section{Physiotherapy intervention}

Fourteen out of 18 players followed the same postoperative protocol, progressed by the treating physiotherapist depending on pain, swelling and of range of movement (ROM). Of the remaining four players, three followed an altered protocol because of them not having access to an AlterG antigravity treadmill (Alter-G, Fremont, California, USA). The final player had a fibula ORIF, which delayed his rehabilitation.

Home exercises were given from week 2 onwards, mirroring progression in the protocol and to supplement daily physiotherapy sessions. Authors AB and BS developed the protocol using the double tightrope repair's ability to progress rehabilitation faster than a traditional screw fixation would allow.

\section{Week 1}

This period included 5 days in a plaster-of-paris (POP) boot postoperatively and 3 weeks in an Aircast boot (DJO Global, Vista, California, USA), progressively increasing weight-bearing as pain and swelling allowed. The Aircast boot was removed for physiotherapy.

\section{Weeks 2 to 4}

The Protection, Rest, Ice, Compression and Elevation (PRICE) principles were followed every 2 hours to reduce swelling and alleviate pain. ${ }^{16}$ The boot was removed for ankle ROM exercises in the sagittal plane only to avoid stressing the repair. Isometric strengthening exercises in plantar grade and foot intrinsic muscle exercises were performed. Proprioception exercises with the boot on and non-weight-bearing exercises to maintain the larger lower limb muscles (gluteals, quadriceps and hamstrings) began.

\section{Weeks 3 to 4}

The boot was removed to allow static bike use as pain allowed. Ankle ROM exercises in all directions (with caution not to force the movement) and isotonic strengthening in the sagittal plane from plantar grade into plantar flexion were also commenced. The PRICE regimen continued every 2 hours.

\section{Week 4}

Following surgical review, the boot was removed and players allowed to run using an AlterG antigravity treadmill at $50 \%$ of body weight providing they were within $2 \mathrm{~cm}$ of their preinjury knee to wall test scores. This was progressed individually according to pain, swelling and restoration of ankle dorsiflexion.

\section{Week 5}

Ankle ROM exercises in all directions were progressed into stiffness with caution not to push into combined dorsiflexion and eversion; this maximally stresses the ankle mortise and the repair. ${ }^{26}$ Manual therapy directed to the superior tibiofibular joint, midfoot and tarsal bones was introduced if necessary.

\section{Week 6}

Exercises were progressed into full weight-bearing with land-based running replacing the use of AlterG antigravity treadmill. Global strengthening of the lower limb in weight-bearing commenced, good hip extension control was encouraged before advancing running. ${ }^{29}$ Subtalar joint mobilisations commenced for appropriate patients, and ROM was pushed into all directions specifically into restricted ranges.

\section{Week 7}

This week included sports-specific running drills, with functional and plyometric exercises. Multiplane exercises to improve ROM and ballistic proprioception 
training were progressed. Manual therapy to the talocrural joint were added to improve dorsiflexion as necessary. ${ }^{30}$ PRICE was encouraged after every training session and after home exercises.

Return to participation (RTP) decisions were made by the physiotherapist. The definition of RTP was return to full participation in training and match play. ${ }^{31}$ It was based on the criteria: ankle dorsiflexion back to pre-injury level (as per preseason testing or compared with uninjured ankle) or $2 \mathrm{~cm}$ or less on the knee to wall test ${ }^{32}$; symmetrical lower limb muscle strength; the ability to perform a symmetrical single leg hop for height and length and the ability to perform the star excursion balance test to pre-injury levels. $^{31} 33$

Three players followed a modified postoperative rehabilitation as they did not have access to an AlterG antigravity treadmill. They were allowed to perform pain-free partial weight-bearing from the outset. The Aircast boot was removed when the subject reported mobilising full weight-bearing, pain free for a whole week. They waited until they could perform a pain-free heel raise, lunge and hop prior to returning to landbased running (two subjects started at 4 weeks with the other waiting until 7 weeks). This did not affect their RTP (mean $=59$ days, SD 12, range 48-72).

One player, due to a fibula ORIF, spent 6 weeks nonweight-bearing in a POP followed by 4 weeks in an Aircast boot. He began running at $50 \%$ body weight on an AlterG antigravity treadmill from 7 weeks and then followed the usual rehabilitation.

Eleven subjects $(61 \%)$ regained full ROM prereturn to sport while seven subjects $(39 \%)$ returned to sport prior to regaining full ROM. This suggests restoration of full ROM is not essential for return to competitive sport and training; this is common in syndesmotic injuries as up to $36 \%$ of cases can have persistent stiffness following injury. ${ }^{26}$

\section{Follow-up}

The average RTP for the whole group was 64 days (SD 17.2, range 38-108). A concomitant ATFL repair did not slow RTP (64 days). The player requiring an ORIF took longer to return to play (108 days RTP). Omitting this players' data, the RTP average for the group reduces to 61.4 days.

Player complaints included: scar infections $(n=2)$; persistent ankle stiffness $(n=2)$; posterior calf tightness $(n=1)$, hip pain $(n=1)$ on return to running; and medial button site irritation $(n=1)$, a problem previously reported in the literature for this procedure. ${ }^{21}$ This player required a guided corticosteroid injection 6 weeks postoperatively, returning to sport after 65 days.

One player did not adhere to his weight-bearing status early in his rehabilitation. This required counselling from the surgeon and a further week in the Aircast boot, subsequently delaying partial weight-bearing running from starting. This did not delay the player's overall RTP time of 68 days.

\section{CONCLUSIONS}

This case series describes a tightrope surgical approach and accelerated rehabilitation protocol following ankle syndesmosis injury. Jelinak and Porter ${ }^{16}$ suggest, following screw fixation, a professional athlete should expect to take between 120 and 180 days to return to sport. Hunt et $a l^{8}$ suggested 70-84 days is the likely RTP time frame with their preferred method of suture button fixation. This case series suggests return to sport is possible within 61 days following syndesmosis injury, provided surgery and rehabilitation is uncomplicated. In instances where there are complications, such as concomitant fibula fracture requiring ORIF, RTP is approximately 103 days. The observed RTP time for uncomplicated syndesmosis repair using this accelerated protocol is an important reduction for professional athletes.

The tightrope approach used in the current case series resulted in few complications. Previous studies have reported complications such as, osteolysis, wound infection, syntosis, heterotopic ossification and a prominent lateral knot leading to soft tissue irritation. ${ }^{3}$ This latter complication is the most commonly cited and can be avoided by adopting a modified surgical technique. ${ }^{34}$ Complication rates range from $8 \%$ to $25 \% .^{3} 35$ 36 What is not clear from previous work is when the complications were discovered, by whom and during what process, for example by the patient at work, by the physiotherapist during rehabilitation or by the surgeon on review.

Delayed diagnosis is associated with prolonged RTP and poor outcomes emphasising the importance of early detection. ${ }^{47}$ The time from initial injury to diagnosis was 1.8 days in this case series (allowing for atypical cases). The diagnosis of syndesmotic injuries is reported to be difficult due to the relative lack of understanding of the condition. ${ }^{5}$ The European Society of Sports Traumatology, Knee Surgery and Arthroscopy-Ankle and Foot Associates (ESSKA-AFAS) recommends the clinical tests: tenderness on palpation over the anterior tibiofibular ligament, the fibular translation test and the Cotton test. ${ }^{38}$ Those used in the current study included ability to hop, tenderness to palpation over the syndesmosis, external rotation test and the squeeze test, as suggested by Smam et al. ${ }^{10}$ In all cases, the injury was observed by medical professionals.

While a battery of physical tests aimed at stressing the ankle syndesmosis can be useful in aiding diagnosis, knowing the mechanism of injury is paramount in determining whether to suspect syndesmotic injury or not. Although using this as the only mechanism to diagnose a syndesmosis injury can be unreliable, and being guided by site of pain, reports of instability, difficulty weight-bearing and inability to push off the toes during gait is more reliable. ${ }^{7}$ In the current case series, 
the mechanism of injury was forced dorsiflexion/eversion $(n=8)$, isolated eversion $(n=2)$, forced rotation $(n=2)$, forced plantar flexion/inversion $(n=3)$, insidious $(n=2)$ and $n=1$ incident of forced plantar flexion. Time from diagnosis to surgery was also short in this group. This was primarily due to the elite nature of the cohort with direct access to an orthopaedic surgeon specialising in syndesmotic injuries. There is currently no evidence as to the optimum time between injury and surgical repair, however, many studies agree that early management provides the best outcome. ${ }^{4} 7839$

\section{Strengths and limitations}

Data collection for each player was without any omissions. The author was independent and had no competing interests towards the findings, and as such, bias should not have been introduced.

The questions used to collect data from clinicians were informed by the available evidence and a process of content validity. The author critically appraised the literature and discussed possible questions with the treating surgeon and physiotherapist involved in developing the rehabilitation protocol. This ensured methodological rigour was maintained to further support the findings.

The cases in this study were heterogeneous. All cases were male, elite level rugby league players with similar ages, levels of fitness, motivation and access to therapy and equipment. The surgery for all cases was carried out by the same surgeon.

Limitations of the study are first, as a retrospective case series, causal relationships cannot be confirmed. ${ }^{40}$ Second, subjects analysed came from six different RFL Super league clubs with slightly different postoperative protocols. Although all clubs had access to the same surgeon, the medical teams, including therapy input will have had varying diagnosis and treatment protocols, which could have significantly influenced results, including RTP times.

\section{Recommendations for future study}

A fully powered randomised controlled trial would determine whether this tightrope repair and accelerated rehabilitation is more effective than the traditional method of syndesmosis repair and rehabilitation as both are currently used.

The RTP time observed in the current case series is comparable to the RTP time of conservatively managed lower grade syndesmotic injuries. ${ }^{13} 30$ Two studies ${ }^{4} 9$ have suggested a lower threshold for surgery in this population and always recommend surgical repair of grade II and above injuries. It provides a dilemma for players and medical professionals alike when dealing with West Point grade II injuries. A comparison of the two approaches would determine whether tightrope repair and accelerated rehabilitation is more beneficial than conservative treatment in grade II injuries.
Prospective longitudinal studies observing the natural course of recovery might help classify syndesmotic injuries over and above those suggested by the ESSKA-AFAS of stable or unstable. ${ }^{38}$ Further classification would be valuable in determining the grade of injury according to severity and aid prognosis and management planning. Causal relationships and predictions between preseason status, previous injury, training and classification or outcome postsurgery, including RTP, could be determined.

All but three players began running on an AlterG antigravity treadmill at $50 \%$ body weight. Interestingly, the three players who did not use the AlterG antigravity treadmill returned to sport slightly quicker than those that did (59 vs 64 days). It is not clear whether the use of the AlterG antigravity treadmill was directly related to RTP. The use and evaluation of AlterG antigravity treadmills in rehabilitation is in its infancy. Evaluations of their effectiveness in the rehabilitation of lower limb injuries, including syndesmosis injury, are scarce and require further investigation.

Contributors BS and PCG conceived the idea for the study. AB and BS developed the clinical protocols. AJL collected the data and provided the background information on syndesmosis repair. PCG provided data analysis. All authors contributed to write-up, editing and approved the final manuscript.

Competing interests None declared.

Ethics approval Manchester Metropolitan University.

Provenance and peer review Not commissioned; externally peer reviewed.

Open Access This is an Open Access article distributed in accordance with the Creative Commons Attribution Non Commercial (CC BY-NC 4.0) license, which permits others to distribute, remix, adapt, build upon this work noncommercially, and license their derivative works on different terms, provided the original work is properly cited and the use is non-commercial. See: http:// creativecommons.org/licenses/by-nc/4.0/

(C) Article author(s) (or their employer(s) unless otherwise stated in the text of the article) 2017. All rights reserved. No commercial use is permitted unless otherwise expressly granted.

\section{REFERENCES}

1. Hermans JJ, Beumer A, de Jong TA, et al. Anatomy of the distal tibiofibular syndesmosis in adults: a pictorial essay with a multimodality approach. J Anat 2010;217:633-45.

2. Warner SJ, Fabricant PD, Garner MR, et al. The measurement and clinical importance of syndesmotic reduction after operative fixation of rotational ankle fractures. J Bone Joint Surg Am 2015:97:1935-44.

3. Al-azzani WAK, Sabah T, Paringe V, et al. Evaluation of ankle tightrope syndesmosis fixation. J Foot Ankle Surg Asia Pacific 2014;1:1-4.

4. Mak MF, Gartner L, Pearce CJ. Management of syndesmosis injuries in the elite athlete. Foot Ankle Clin 2013;18:195-214.

5. Thormeyer JR, Leonard JP, Hutchinson M. Syndesmotic injuries in athletes, an international perspective on topics in sports medicine and sports injury. In: Kenneth D, Zaslav R, InTech. ed, 2012. ISBN: 978-953-51-0005-8. http://www.intechopen.com/books/an-international-perspectiveon-topics-in-sports-medicine-and-sports-injury/ syndesmotic-injuries-in-athlete.

6. Miller TL, Skalak T. Evaluation and treatment recommendations for acute injuries to the ankle syndesmosis without associated fracture. Sports Med 2014;44:179-88.

7. Hsu AR, Garras DN, Lee S. Syndesmotic injuries in athletes. Oper Tech Sports Med 2014;22:270-81. 
8. Hunt KJ, Phisitkul P, Pirolo J, et al. High ankle sprains and syndesmotic injuries in athletes. J Am Acad Orthop Surg 2015;23:661-73.

9. Porter DA, Jaggers RR, Barnes AF, et al. Optimal management of ankle syndesmosis injuries. Open Access J Sports Med 2014;5:173-82.

10. Sman $A D$, Hiller $C E$, Rae $K$, et al. Diagnostic accuracy of clinical tests for ankle syndesmosis injury. Br J Sports Med 2015;49:323-9.

11. Wright RW, Barile RJ, Surprenant DA, et al. Ankle syndesmosis sprains in national hockey league players. Am J Sports Med 2004;32:1-5.

12. Gerber JP, Williams GN, Scoville CR, et al. Persistent disability associated with ankle sprains: a prospective examination of an athletic population. Foot Ankle Int 1998;19:653-60.

13. Sman AD, Hiller $C E$, Rae $K$, et al. Prognosis of ankle syndesmosis injury. Med Sci Sports Exerc 2014;46:671-7.

14. Cottom JM, Hyer CF, Philbin TM, et al. Transosseous fixation of the distal tibiofibular syndesmosis: comparison of an interosseous suture and endobutton to traditional screw fixation in 50 cases. $J$ Foot Ankle Surg 2009;48:620-30.

15. Sagi HC, Shah AR, Sanders RW. The functional consequence of syndesmotic joint malreduction at a minimum 2-year follow-up. $J$ Orthop Trauma 2012;26:439-43.

16. Jelinek JA, Porter DA. Management of unstable ankle fractures and syndesmosis injuries in athletes. Foot Ankle Clin 2009;14:277-98.

17. Naqvi GA, Shafqat A, Awan N. Tightrope fixation of ankle syndesmosis injuries: clinical outcome, complications and technique modification. Injury 2012;43:838-42.

18. Qamar F, Kadakia A, Venkateswaran B. An anatomical way of treating ankle syndesmotic injuries. J Foot Ankle Surg 2011;50:762-5

19. Konor MM, Morton S, Eckerson JM, et al. Reliability of three measures of ankle dorsiflexion range of motion. Int J Sports Phys Ther 2012;7:279-87.

20. Takao M, Ochi M, Oae K, et al. Diagnosis of a tear of the tibiofibular syndesmosis. J Bone Joint Surg 2003;85:324-9.

21. Naqvi GA, Shafqat A, Awan N. Tightrope fixation of ankle syndesmosis injuries: clinical outcome, complications and technique modification. Injury 2012;43:838-42.

22. Qamar F, Kadakia A, Venkateswaran B. An anatomical way of treating ankle syndesmotic injuries. J Foot Ankle Surg 2011;50:762-5.

23. Rigby RB, Cottom JM. Does the arthrex TightRope $\AA$ provide maintenance of the distal tibiofibular syndesmosis? A 2-year followup of 64 TightRopes $\mathbb{R}$ in 37 patients. J Foot Ankle Surg 2013;52:563-7.

24. Naqvi GA, Cunningham $P$, Lynch $B$, et al. Fixation of ankle syndesmotic injuries: comparison of tightrope fixation and syndesmotic screw fixation for accuracy of syndesmotic reduction. Am J Sports Med 2012;40:2828-35.
25. Willmott HJ, Singh B, David LA. Outcome and complications of treatment of ankle diastasis with tightrope fixation. Injury 2009;40:1204-6.

26. Mulligan EP. Evaluation and management of ankle syndesmosis injuries. Phys Ther Sport 2011;12:57-69.

27. Gagnier JJ, Kienle G, Altman DA, et al. The CARE group. The CARE guidelines: consensus-based clinical case reporting guideline development. Global Adv Health Med 2013;2:38-43.

28. Sikka RS, Fetzer GB, Sugarman $\mathrm{E}$, et al. Correlating MRI findings with disability in syndesmotic sprains of NFL players. Foot Ankle Int 2012;33:371-8

29. Comerford MJ. Screening to identify injury and performance risk: movement control testing- the missing piece of the puzzle. Sport Ex Medicine 2006:21-6.

30. Morgan C, Kanopinski M, Dunn A. Conservative management of syndesmosis injuries in elite football- an injury on the increase or an increase in recognition. Sports Med J 2014:602-13.

31. Fuller CW, Molloy MG, Bagate C, et al. Consensus statement on injury definitions and data collection procedures for studies of injuries in rugby union. Clin J Sport Med 2007;17:177-81.

32. Konor MM, Morton S, Eckerson JM, et al. Reliability of three measures of ankle dorsiflexion range of motion. Int J Sports Phys Ther 2012;7:279-87.

33. Gribble PA, Hertel J, Plisky P. Using the star excursion balance test to assess dynamic postural-control deficits and outcomes in lower extremity injury: a literature and systematic review. J Athl Train 2012:47:339-57.

34. Hodgson P, Thomas R. Avoiding suture knot prominence with suture button along distal fibula: technical tip. Foot Ankle Int 2011;32:908-9.

35. Storey P, Gadd RJ, Blundell C, et al. Complications of suture button ankle syndesmosis stabilization with modifications of surgical technique. Foot Ankle Int 2012;33:717-21.

36. Degroot H, Al-Omari AA, El Ghazaly SA. Outcomes of suture button repair of the distal tibiofibular syndesmosis. Foot Ankle Int 2011;32:250-6.

37. Wolf BR, Amendola A. Syndesmosis injuries in the athlete: when and how to operate. Curr Opin Orthop 2002;13:151-4.

38. van Dijk CN, Longo UG, Loppini M, et al. Classification and diagnosis of acute isolated syndesmotic injuries: esska-afas consensus and guidelines. Knee Surg Sports Traumatol Arthrosc 2016;24:1200-16.

39. Kortekangas T, Savola O, Flinkkilä T, et al. A prospective randomised study comparing TightRope and syndesmotic screw fixation for accuracy and maintenance of syndesmotic reduction assessed with bilateral computed tomography. Injury 2015;46:1119-26.

40. Hicks C. Research methods for clinical therapists. 4th edn. Edinburgh: Churchill Livingstone, 2004. 